\title{
Optimization of Sheet Metal Forming Process Parameters by Artificial Neural Network and Orthogonal Test Method
}

\author{
Wenqiong Zhang and Dongwei Wang \\ School of electrical and Mechanical Engineering, Xiamen University of Tan Kah Kee, Xiamen 363105, China
}

\begin{abstract}
Combined with artificial neural network of good features, using the orthogonal experiment data obtained as the training sample of neural network we established a neural network model of the input for the process parameters, output for the springback amount, and the accuracy of the ANN model was verified by the sample, so as to shorten the time of setting the process parameters. Within the scope of the process parameter selection, the ANN model instead of CAE software simulation test, combined with orthogonal experiment method, to further optimize the process parameters. Results show that the neural network combined with orthogonal test, numerical simulation was applied to parameter optimization of sheet metal forming, can shorten the time of the optimization of process parameters, and improve the efficiency of process design.
\end{abstract}

Keywords-Artificial Neural Network (ANN); orthogonal experiment; technological parameter; optimization

\section{INTRODUCTION}

As one of the basic methods of metal plastic processing, sheet metal forming is widely used in aerospace, automobile, home appliance and other industries, and has a very important position in the industrial production. With the development of finite element technology, using numerical simulation we can accurately predict the results of sheet metal forming, but in the optimization of forming process parameters, still mainly use the numerical simulation combined with "trial and error" method to do so. Namely according to the results of numerical simulation, and the experience of modified process parameters, design personnel carry out the simulation [1-2].But the selection of process parameters involves thousands of combinations, and in the presence of numerous combinations, it is difficult to get the optimal parameter combination by experience. So we used artificial neural network, orthogonal experiment and numerical simulation technology for the sheet metal forming parameters optimization.

\section{The ESTABLISHMENT AND TRAINING OF ANN MODEL}

Artificial neural network (ANN) is a complex network system, which is composed of a large number of simultaneous and very simple processing units (or neurons). With massively parallel distributed storage and processing, adaptive and selflearning capabilities, it is especially suitable for processing need to consider many factors and conditions, inaccurate and fuzzy information problems [3]. The BP network is a network model which is widely used at present. It has the functions of self organization, self-learning, associative memory, and other functions, and its fault-tolerant performance is also very good. In the case of sufficient hidden layer neurons, the BP network with a deviation, with at least one $\mathrm{S}$ type hidden layer and a linear output layer can approximate any complex nonlinear function [4]. It needs to deal with a lot of raw data to establish input for forming process parameters, output for the springback amount of mapping relation model, and these data are hard to use a unified formula or rule describing, cannot be expressed in mathematical model. Therefore, the artificial neural network is a favorable tool to solve the problem with its great flexibility and adaptability.

\section{A. The Determination of Network Input and Output Parameters}

Input and output parameters is the key link in the process of establish a neural network. Parameter selection should be both response to the imitating the behavior of the system pattern, and concise. For output prediction model of springback amount $\Delta Z$, due to the many factors that affect the resilience, in the actual mold design and production technology, the process and die parameters are the most easy to control, although parts of complexity and also on springback of a very important influence, but they are not considered here for convenience of simulation analysis. In this paper, the influence factors to be considered are: blank-holder force $F$, die clearance $\lambda$, friction coefficient ${ }^{\mu}$, stamping speed $V$, drawbead (force) $L$.

\section{B. The Selection of Training Samples}

Orthogonal design is a scientific arrangement and analysis method of multi-factor experiments, it is from the comprehensive test to pick out part of the uniform, neat, representative test points, is the main method of fractional factorial design, with very high efficiency. Using orthogonal design method we can solve multi - factor, multi - level and multi - index test question. Orthogonal design, although a lot of test times reduced, is obviously able to determine the primary and secondary influence factors, the relationship between factors and indicators, the merits of the production conditions and the direction of further testing and other issues [5].

The orthogonal test method has the characteristic of the balanced dispersion and the neat and comparable, and it can reflect the overall situation with less experiment [6]. Therefore, the main purpose of this paper is using the method of 
orthogonal experiment to determine the distribution of training samples; in addition, according to the size of the different variables influence on springback, properly adjust the distribution of some samples, such as for the variables of relatively bigger influence, to increase some of the value of the sample. In this paper, the back wall stamping of a tractor is considered as an example, its material is 08 steel, the thickness of the plate is $1.5 \mathrm{~mm}$. With the sheet metal forming CAE analysis software DYNAFORM simulation experiments we obtained different sheet metal forming process parameters and its springback amount after forming, the samples for the orthogonal experiment and numerical simulation of the 16 groups of experimental data, as shown in table 1.

\section{The Determination of Network Structure and Training Method}

a) Network of 3 layers, input layer with 5 nodes, hidden layer with 16 nodes, 1 nodes in the output layer.

b) The transfer function between the input and the hidden layer using the Tansig transfer function; between the hidden layer and output layer using linear Tansig function.

c) The network training function using the conjugate gradient algorithm is good for large-scale network training effect, and the training speed is fast.

d) The training deviation: the comparison deviation between ANN training results and the training sample data was controlled within $1 \%$, the rebound analysis for the selected workpiece has meet the accuracy requirements.

TABLE I. THE TRAINING SAMPLE OF NEURAL NETWORKF

\begin{tabular}{lcccccc}
\hline No. & $F / \mathrm{KN}$ & $\mu$ & $\lambda / \mathrm{mm}$ & $\nu \mathrm{mm} / \mathrm{s}$ & $L / \mathrm{KN}$ & $\Delta Z / \mathrm{mm}$ \\
\hline 1 & 1300 & 0.07 & 1.65 & 3500 & 60 & 5.356 \\
2 & 1300 & 0.09 & 1.63 & 3000 & 70 & 4.134 \\
3 & 1300 & 0.12 & 1.61 & 4500 & 50 & 5.048 \\
4 & 1300 & 0.14 & 1.59 & 4000 & 80 & 3.121 \\
5 & 1400 & 0.07 & 1.61 & 4000 & 80 & 6.109 \\
6 & 1400 & 0.09 & 1.59 & 3500 & 50 & 3.975 \\
7 & 1400 & 0.12 & 1.65 & 3000 & 60 & 4.153 \\
8 & 1400 & 0.14 & 1.63 & 4500 & 70 & 4.115 \\
9 & 1500 & 0.07 & 1.61 & 4000 & 70 & 4.527 \\
10 & 1500 & 0.09 & 1.65 & 3000 & 50 & 4.379 \\
11 & 1500 & 0.14 & 1.63 & 3500 & 80 & 3.533 \\
12 & 1500 & 0.12 & 1.59 & 4500 & 60 & 3.095 \\
13 & 1600 & 0.07 & 1.63 & 3500 & 60 & 7.761 \\
14 & 1600 & 0.09 & 1.65 & 3000 & 80 & 4.687 \\
15 & 1600 & 0.12 & 1.61 & 4500 & 50 & 5.762 \\
16 & 1600 & 0.14 & 1.59 & 4000 & 70 & 4.048 \\
\hline
\end{tabular}

\section{ANN MODEL TEST}

After the training of neural network, there is a problem, that is, how to test whether the network's rebound results are correct. In this study, a new test data test method has been adopted. That is, first using a group of parameters of the process data to train the neural network, then to retake a group of new technological parameter data, respectively using neural network and numerical simulation method to calculate and predict the springback amount, and t0 compare the two sets of results, we can see whether the neural network prediction results of springback are of versatile in the range of parameters, as shown in Table 2.

TABLE II. COMPARISON OF ARTIFICIAL NEURAL NETWORK PREDICTION RESULTS AND NUMERICAL SIMULATION RESULTS

\begin{tabular}{cccc}
\hline $\begin{array}{c}\text { Test group } \\
\text { number }\end{array}$ & $\begin{array}{c}\text { Prediction results } \\
\text { of neural network }\end{array}$ & $\begin{array}{c}\text { The results of numerical } \\
\text { simulation of springback }\end{array}$ & $\begin{array}{c}\text { Error } \\
(\%)\end{array}$ \\
\hline 1 & 4.396 & 4.256 & 3.301 \\
2 & 3.491 & 3.448 & 1.251 \\
3 & 3.307 & 3.345 & 1.140 \\
4 & 4.667 & 4.789 & 2.545 \\
5 & 4.265 & 4.147 & 2.759 \\
\hline
\end{tabular}

From the table above, you can see that in the parameter range, the spring back prediction results of neural network are very close to the numerical simulation results, which shows that the established model is effective and reliable.

\section{Optimization of Process Parameters Based ON NEURAL NETWORK MODEL}

Hypothesis that the nonlinear mapping relationship from the process parameters to the workpiece springback amount is $\boldsymbol{D}=\boldsymbol{f}\left(\boldsymbol{Y}_{\mathbf{1}}, \boldsymbol{Y}_{\mathbf{2}} \cdots \cdots \boldsymbol{Y}_{\boldsymbol{c}}\right)$, then the best parameter combination of the orthogonal test is the extreme value of the discrete points in $\boldsymbol{D}=\boldsymbol{f}\left(\boldsymbol{x}_{\mathbf{r}}\right)$, there may be got more excellent parameter combination than the orthogonal experiment in the vicinity of the extreme.

\section{A. Optimization Steps}

The using search optimization method of small step way, we would gradually reduce the search scope of process parameter optimization, which concrete steps as follows:

a)Near the optimum point (obtained the best parameter combination from orthogonal test) for each variable process parameters we increase and reduce the tiny step $\delta_{i}(i=1,2 \cdots \cdots 6)$, match these parameters into the combination of multiple sets of parameters, then generate a new orthogonal table.

b) A series of springback amount $\Delta Z$ are calculated by the ANN model.

c) Find the corresponding parameters combination of smaller springback $\Delta Z$.

d) Return 1, until the amount $\Delta Z$ of springback numerical achieved so far.

\section{B. Optimization Process}

For the results of the optimal process parameter combination of the orthogonal experiment, tiny variables were taken in the vicinity, as shown in table 3. 
TABLE III. THE FACTORS AND LEVEL OF OPTIMIZATION

\begin{tabular}{cccccc}
\hline No. & $F / \mathrm{KN}$ & $\mu$ & $\lambda / \mathrm{mm}$ & $\nu \mathrm{mm} / \mathrm{s}$ & $L / \mathrm{KN}$ \\
\hline 1 & 1460 & 0.116 & 1.586 & 3600 & 56 \\
2 & 1680 & 0.118 & 1.588 & 3800 & 58 \\
3 & 1500 & 0.120 & 1.590 & 4000 & 60 \\
4 & 1520 & 0.122 & 1.592 & 4200 & 62 \\
\hline
\end{tabular}

Also using orthogonal table to arrangement the experiment, and using ANN model to get the springback amount, the results as shown in table 4.

A better process parameters combination still can been found by the neural network in the vicinity of the orthogonal experiment and the optimal process parameters combination, the combination of blank-holder force $1500 \mathrm{KN}$, friction coefficient of 0.118, convex concave die clearance of 1.592 $\mathrm{mm}$, stamping speed $3600 \mathrm{~mm} / \mathrm{s}$, drawbead $56 \mathrm{KN}$ (power), amount of springback $2.653 \mathrm{~mm}$ calculated by the ANN model.

\section{TEST OPTIMAL SOLUTION}

In order to examine the ANN model whether the optimal solution meet the CAE simulation software, compared with the results by the sheet metal forming finite element software DYNAFORM, the springback amount of artifacts is $2.743 \mathrm{~mm}$, the springback amount of error is $3.415 \%$, namely the mapping artificial neural network for establishing the parameters of springback quantity is basic accurate. In search of extreme existing unstable phenomenon, it is also proved that the artificial neural network in parameter prediction and optimization is applicable.

TABLE IV. TRAINING RESULT

\begin{tabular}{ccccccc}
\hline No. & $F / \mathrm{KN}$ & $\mu$ & $\lambda / \mathrm{mm}$ & $v \mathrm{~mm} / \mathrm{s}$ & $L / \mathrm{KN}$ & $\Delta Z / \mathrm{mm}$ \\
\hline 1 & 1460 & 0.116 & 1.592 & 3800 & 58 & 3.345 \\
2 & 1460 & 0.118 & 1.590 & 3600 & 60 & 2.749 \\
3 & 1460 & 0.120 & 1.688 & 4200 & 56 & 3.448 \\
4 & 1460 & 0.122 & 1.586 & 4000 & 62 & 3.124 \\
5 & 1480 & 0.116 & 1.588 & 4000 & 62 & 2.849 \\
6 & 1480 & 0.118 & 1.586 & 3800 & 56 & 3.086 \\
7 & 1480 & 0.120 & 1.592 & 3600 & 58 & 3.364 \\
8 & 1480 & 0.122 & 1.590 & 4200 & 60 & 3.002 \\
9 & 1500 & 0.116 & 1.588 & 4200 & 60 & 3.179 \\
10 & 1500 & 0.118 & 1.592 & 3600 & 56 & 2.653 \\
11 & 1500 & 0.120 & 1.590 & 3800 & 62 & 2.975 \\
12 & 1500 & 0.122 & 1.586 & 4000 & 58 & 3.127 \\
13 & 1520 & 0.116 & 1.590 & 3800 & 58 & 3.205 \\
14 & 1520 & 0.118 & 1.592 & 3600 & 62 & 2.875 \\
15 & 1520 & 0.120 & 1.588 & 4200 & 56 & 2.946 \\
16 & 1520 & 0.122 & 1.586 & 4000 & 50 & 3.030 \\
\hline
\end{tabular}

\section{ACKNOWLEDGMENT}

Through the above analysis shows that the combination of neural network and orthogonal experiment, numerical simulation for sheet metal forming parameters optimization can significantly shorten the time to optimize the process parameters, improve the efficiency of process design, and can obtain more optimal results than the simple use of the orthogonal
Support project: education and scientific research projects of young and middle-aged teachers in Fujian Province

\section{REFERENCES}

[1] Li Yuqiang, Cui Zhenshan, Zhang Dongjuan, et al. Research on the optimization of sheet metal forming process and quality engineering $[\mathrm{J}]$. Journal of plastic engineering, 2005,12 (2): 11-16.

[2] Pan Jiangfeng, clock about chao-long yuan, etc. The sheet metal forming process parameters of multi-objective genetic algorithm optimization [J]. Journal of Tsinghua University: Natural Science Edition, 2007, 47 (8): 1267-1269.

[3] Research and development center of science and technology products. The neural network theory and MATLAB7 realize [M]. Beijing: electronic industry press, 2005.

[4] Dong Xiaolan Wu Wei, etc. The BP neural network and its application in optimum design of die casting mould is [J]. Journal of mould technology, 2006, 44 (6): 6-10.

[5] Zhang Chao, etc. The orthogonal experiment method application in the automobile fender forming technology [J]. Journal of mold industry, 2007, 33 (5) : 26-29.

[6] Gao Jun, Zhang Chengrui, Ji Tingwei, et al. The selection method of neural network training samples based on orthogonal design [J]. Journal of plastic engineering, 2006,13 (5): 32-35. 